\title{
A randomized controlled trial of an intervention delivered by mobile phone app instant messaging to increase the acceptability of effective contraception among young people in Tajikistan
}

Ona McCarthy ${ }^{1 *}$, Irrfan Ahamed ${ }^{1}$, Firuza Kulaeva², Ravshan Tokhirov², Salokhiddin Saibov², Marieka Vandewiele ${ }^{3}$, Sarah Standaert ${ }^{3}$, Baptiste Leurent ${ }^{4}$, Phil Edwards ${ }^{1}$, Melissa Palmer ${ }^{1}$ and Caroline Free ${ }^{1}$

\begin{abstract}
Background: Unintended pregnancy is associated with poorer health outcomes for women and their families. In Tajikistan, around 26\% of married 15-24 year old women have an unmet need for contraception. There is some evidence that interventions delivered by mobile phone can affect contraceptive-related behaviour and knowledge. We developed an intervention delivered by mobile phone app instant messaging to improve acceptability of effective contraceptive methods among young people in Tajikistan.

Methods: This was a randomized controlled trial among Tajik people aged 16-24. Participants allocated to the intervention arm had access to an app plus intervention messages. Participants allocated to the control arm had access to the app plus control messages. The primary outcome was acceptability of at least one method of effective contraception at 4 months. Secondary outcomes were use of effective contraception at 4 months and during the study, acceptability of individual methods, service uptake, unintended pregnancy and induced abortion. Process outcomes were knowledge, perceived norms, personal agency and intention. Outcomes were analysed using logistic and linear regression. We conducted a pre-specified subgroup analysis and a post-hoc analysis of change in acceptability from baseline to follow-up.

Results: Five hundred and seventy-three participants were enrolled. Intervention content was included on the app, causing contamination. Four hundred and seventy-two (82\%) completed follow-up for the primary outcome. There was no evidence of a difference in acceptability of effective contraception between the groups (66\% in the intervention arm vs $64 \%$ in the control arm, adjusted OR $1.21,95 \% \mathrm{Cl} .80-1.83, p=0.36$ ). There were no differences in the secondary or process outcomes between groups. There was some evidence that the effect of the intervention was greater among women compared to men (interaction test $p=0.03$ ). There was an increase in acceptability of effective contraception from baseline to follow-up $(2 \%$ to $65 \%, p<0.001)$.

(Continued on next page)
\end{abstract}

\footnotetext{
* Correspondence: ona.mccarthy@lshtm.ac.uk

${ }^{1}$ Department of Population Health, Faculty of Epidemiology and Population

Health, London School of Hygiene \& Tropical Medicine, Keppel Street,

London WC1E 7HT, UK

Full list of author information is available at the end of the article
} 
(Continued from previous page)

Conclusions: The whole intervention delivered by instant messaging provided no additional benefit over a portion of the intervention delivered by app pages. The important increase in contraceptive acceptability from baseline to follow-up suggests that the intervention content included on the app may influence attitudes. Further research is needed to establish the effect of the intervention on attitudes towards and use of effective contraception among married/sexually active young people.

Trial registration: Clinicaltrial.gov NCT02905513. Date of registration: 14 September 2016.

Keywords: Randomized controlled trial, Tajikistan, Contraception, Smart phone, Reproductive health, Young adults

\section{Plain English summary}

Unintended pregnancy is associated with poor health and social outcomes for women and their families. Despite wide availability of contraception, many women globally face barriers in realizing their fertility desires. A woman has an unmet need for modern contraception if she wants to avoid a pregnancy but currently uses no method or a traditional method. In Tajikistan, unmet need for contraception is approximately $26 \%$ among married 15-24 year olds. Oppositional attitudes towards contraception (both their own and others') is a common reason women provide for not using contraception.

We developed an intervention delivered by mobile phone to increase the acceptability of effective contraception among young people in Tajikistan. The intervention was developed with young people using an established approach grounded in behavioural science. We conducted a randomized controlled trial to evaluate the effect of the intervention on acceptability of effective contraception. Participants allocated to the intervention group had access to an app plus the intervention messages. Participants allocated to the control group had access to the app plus control messages. The app contained a proportion of the intervention messages that targeted knowledge of and attitudes towards effective contraception. This was different from what was planned in the trial protocol.

The intervention instant messages did not have an added benefit over the app with regards to any of the outcomes. When data from both groups were analysed together, there was a large increase in acceptability of effective contraception from baseline to follow-up ( $2 \%$ at baseline to $65 \%$ at follow-up). While we cannot attribute this increase unequivocally to the intervention content, it suggests that providing accurate information and targeting beliefs that influence contraceptive use may be sufficient in changing attitudes towards these methods among young people in Tajikistan. Further research is needed to reliably establish the effect of the intervention on attitudes towards and use of effective contraceptive methods among married/sexually active young people.

\section{Background}

Unintended pregnancy persists as a global health problem, with people in lower income countries experiencing them at a higher rate [1]. Unintended pregnancy is associated with a multitude of negative health and economic outcomes for women and their families [2-11]. It is estimated that modern contraceptive use currently prevents 307 million unintended pregnancies each year in developing regions [12]. Satisfying unmet need for modern contraception in these regions would reduce unintended pregnancies by $74 \%$ [12]. A woman has an unmet need for modern contraception if she wants to avoid a pregnancy but currently uses no method or a traditional method [13].

Despite a number of governmental policy initiatives and strategies aimed at improving reproductive health in Tajikistan, young people in the country face challenges in gaining accurate information about contraception and in accessing services [14, 15]. The 2012 Tajikistan Demographic and Health Survey is the most reliable resource for family planning data in the country at present [16]. The survey estimates that Tajik women have an average of half a child more than their desired number, implying that if unintended pregnancies were avoided, the total fertility rate would be 3.3 births per woman rather than the actual 3.8 [16]. The effective contraceptive methods available in Tajikistan are oral contraceptive pills (OCs), intrauterine devices (IUDs), injectables and implants ('effective methods are methods with a less than $10 \%$ typical use failure rate at 12 months [17-19]). Though these methods are available, around $26 \%$ of married 15-24 year old women have an unmet need for contraception [16]. Unmet need is the highest between the ages of 20 to 29 [20]. The main reason women with an unmet need provide for not using contraception are oppositional attitudes towards contraception, both their own and others' [20]. The next common reasons relate to low perceived pregnancy risk and negative attitudes about the methods, such as fear of side-effects [20].

Over the past few decades, the dramatic global increase in mobile phone ownership has engendered enthusiasm amongst researchers and health care 
providers regarding the use of mobile phones for health care delivery [21-32]. Trials have provided some evidence that interventions delivered by mobile phone can improve contraceptive-related behaviours [33-36] and knowledge [37-39], however others have failed to find an effect [40-43]. The London School of Hygiene and Tropical Medicine (LSHTM) and the Tajik Family Planning Association (TFPA), a Member Association of the International Planned Parenthood Federation (IPPF) collaborated to develop and evaluate an intervention delivered by mobile phone to improve attitudes towards the effective contraceptive methods among young people in Tajikistan.

To evaluate the intervention, we conducted a randomized controlled trial from November 2016 to July 2017. This paper reports the results of the trial. To the best of our knowledge, this is the first trial to evaluate a contraceptive behavioural intervention delivered by mobile phone in Tajikistan. The results contribute to an understanding about how to help young people in Tajikistan avoid unintended pregnancies.

\section{Methods}

The methods reported in this section were first published in the trial protocol [44] and the statistical analysis plan [45].

\section{Study design and participants}

This was a parallel group, individually randomized superiority trial with a 1:1 allocation ratio. The aim of this trial was to assess the effect of the intervention on the acceptability of effective contraceptive methods among young people in Tajikistan. Participants were eligible to take part in the trial if they were between the ages of 16 and 24, owned a personal Android mobile phone, lived in Tajikistan, could provide informed consent and could read Tajik or Russian. Participants must also have been willing to download a mobile phone app and receive instant messages about contraception through the app. Participants provided informed consent though the secure online trial database and randomization system. All participants received usual care (the normal care that a young person would receive if they attended a sexual and reproductive health service in Tajikistan) and were free to seek any other support.

\section{Intervention and control}

The intervention was developed with young Tajik people in 2015-2016 guided by an established approach grounded in behavioural science [46]. It consisted of short mobile phone instant messages delivered through TFPA's 'healthy lifestyles' app over 4 months. It was informed by the Integrated Behavioural Model (IBM) [47] and contained 10 behaviour change methods (BCM) (belief selection, facilitation, anticipated regret, guided practice, verbal persuasion, tailoring, cultural similarity, arguments, shifting perspective and goal setting) [48], adapted for delivery by mobile phone. The messages provided information about contraception, targeted beliefs identified in the development phase that influence contraceptive use and aimed to support young people in believing that they can influence their reproductive health.

The messages are tailored according to marital status and gender, resulting in four sets of messages (femalemarried, female-not married, male-married and malenot married). The majority of the messages in the four sets are the same, with minor tailoring so that the messages are relevant to these groups. (Marital status was used as a proxy for sexual activity because the target group and TFPA considered it inappropriate to ask directly about sexual activity.) Further details about the intervention are presented in the trial protocol [44] and in a forthcoming intervention development publication.

\section{Contamination}

Participants allocated to the intervention arm had access to the app plus the intervention instant messages. Participants allocated to the control arm had access to the app plus control instant messages about trial participation. Contrary to what was planned in the trial protocol [44], the app contained intervention content. The app was intended to contain only basic information about contraception and no behaviour change methods. This contamination occurred due to a misunderstanding between the partners collaborating in the research.

The app contraception pages included just under a third of the intervention content. Specifically, $57 \%$ of the femalemarried intervention messages that provide accurate information about the effective contraceptive methods and $36 \%$ of the messages that use the BCM 'belief selection' were included on the app. Forty-four percent of the femalemarried intervention content included on the app used the same words as the intervention messages (56\% did not use the same words but was very similar and conveyed the same meaning). The intervention content included on the app aimed to help individuals: name the effective methods, describe how the effective methods work, list services that provide effective contraception, list the risks and benefits of the effective methods, describe how methods are used, express positive attitudes towards the effective methods and differentiate between real potential side-effects and misconceptions about the methods.

\section{Allocation and intervention delivery}

After providing informed consent, participants completed the baseline questionnaire through the database 
and randomization system. The allocation sequence was generated by the remote computer-based randomization software. Randomization occurred immediately after baseline data was submitted. All participants downloaded the app immediately after they submitted their baseline data. The delivery of the intervention (and control) instant messages began on the same day if participants downloaded the app before 13:00 and the following day if they downloaded it after 13:00.

\section{Protecting against bias}

Due to the nature of the intervention, participants would have been aware of the allocation soon after they started receiving the messages. Local research staff collecting outcome data were masked to allocation unless the participant revealed it to them. Researchers that analysed the data were masked to treatment allocation.

\section{Outcomes}

\section{Primary outcome}

The primary outcome was the proportion of participants reporting that at least one method of effective contraception was acceptable at 4 months post randomization. The primary outcome measure was constructed based on guidelines for measuring IBM constructs $[47,49,50]$ and tested for face validity with the target group. The acceptability of each method was measured by the following stems: Using the [method] ...causes infertility, ...causes unwanted side effects, ...is easy, ...is a good way to prevent pregnancy and I would recommend the [method] to a friend. The IUD and implant include an additional stem: The [method] insertion would not be a problem. The response options for each scale were strongly disagree, disagree, not sure, agree, strongly agree and I do not know what the [method] is. A method was acceptable if participants reported 'agree' or 'strongly agree' for all scales except for '...causes infertility' and '...causes unwanted side effects' stems, for which 'disagree' or 'strongly disagree' indicated acceptability.

\section{Secondary outcomes}

Secondary outcomes were: use (or partner's use) of effective contraception; acceptability of individual methods; use (or partner's use) of effective contraception at any time during the 4 months; service uptake; unintended pregnancy and induced abortion.

Process outcomes The process outcomes were: knowledge of effective contraception; perceived norms in relation to using and communicating with partners about contraception; personal agency in using (women only) and communicating with partners about contraception; intention to use effective contraception (women only) and intervention dose received. Details about the scales used to measure knowledge, perceived norm, personal agency and intention are reported in the trial protocol [44].

\section{Data collection}

Data was collected at baseline and at 4 months postrandomization using questionnaires. At baseline, we collected personal and demographic data and acceptability of at least one method of effective contraception (using the same scales as the primary outcome measure). All baseline data was entered onto the trial database system by the participant on their mobile phone. At 4 month follow-up, we collected all outcomes and the following data: if participants report using an effective method, where they obtained it; current pregnancy intention; whether they knew someone else that took part in the study and if so, if they read each other's messages; if they stopped the messages; if they experienced physical violence since being in the study and if anything good or bad happened as a result of receiving the messages. An instant message that included a link to the database to complete the follow-up questionnaire was sent to all participants through the app 4 months after downloading the app. If participants did not complete the follow-up questionnaire themselves, local research staff contacted them by telephone to collect their data.

\section{Sample size}

The trial was powered to detect a $15 \%$ increase in acceptability of effective contraception in the intervention group compared with the control group. Four hundred and fifty-four participants allowed for $90 \%$ power to detect a $15 \%$ absolute increase in acceptability, assuming $50 \%$ acceptability in the control group (i.e. $50 \%$ in the control vs $65 \%$ in the intervention, an odds ratio of 1.86). Allowing for $20 \%$ loss to follow-up, we aimed to randomize 570 people.

\section{Statistical analysis}

The trial protocol was accepted for publication on 21 July 2017 [44] and the statistical analysis plan was publicly released on 16 August 2017 [45]. The analysis was conducted using Stata 15. Analyses were according to randomized arm and only participants with complete outcome data were included in the principal analysis. All statistical tests were two-sided and considered significant at the 5\% level. Unmasking occurred on 29 August 2017, after the analyses outlined within the analysis plan were complete. 


\section{Loss to follow-up and missing data}

We used a chi-squared test to investigate whether loss to follow-up differed by arm. We used logistic regression to compare baseline characteristics of participants that completed follow-up against participants that did not. We investigated whether predictors of loss to follow-up differed by arm by testing for an interaction.

\section{Principal analysis}

\section{Analysis of the primary outcome}

We compared the proportion that reported that at least one method was acceptable in each group using logistic regression. We report the crude and adjusted odds ratio (OR) along with the $95 \%$ confidence interval (CI) and $p$ value. We adjusted the primary analysis regression for the following pre-specified baseline covariates: pregnancy intention (wants to avoid/other); gender (female/male); age (16-19/20-24); highest education level completed (university/other) and acceptability of effective contraception (at least one method acceptable/ no methods acceptable) [44, 45].

\section{Analysis of the secondary outcomes}

The analysis of the secondary outcomes was similar to the analysis of the primary outcome. We estimated the difference between the groups using logistic regression and report odds ratios with $95 \%$ CIs and $p$-values. Regressions were adjusted for the baseline covariates pregnancy intention, gender, age, education level and acceptability (of at least one method or with acceptability of individual methods, of the corresponding method).

\section{Analysis of the process outcomes}

The process outcomes perceived norms, personal agency and intention were comprised of ordinal scales. Each scale was analysed individually using ordered logistic regression to estimate proportional ORs. For knowledge, each correct answer received one point. The points were summed and an overall score was produced. We used linear regression to test for a difference in mean scores between the arms. To assess the 'dose' of the intervention that the intervention participants received, we analysed the number of messages that participants reported to have read (all, most, some, none) and whether they stopped the messages.

\section{Additional analyses}

\section{Sensitivity analyses}

We conducted two sensitivity analyses regarding the missing data. In the first, we considered that participants lost to follow-up did not find at least one method acceptable. In the second, we adjusted for the main baseline predictors of missingness. Both sensitivity analyses were adjusted for the baseline covariates pregnancy intention, gender, age, education level and acceptability.

\section{Subgroup analysis}

We conducted an exploratory subgroup analysis for the primary outcome to determine if the intervention effect varied by baseline characteristics. The prespecified subgroups were gender (female/male); age (split at the median); marital status (married/not married); number of children $(0 / 1+)$; ethnicity (Tajik/ other); occupation (in education/other); highest education level completed (university/other) and pregnancy intention (wants to avoid/other). Within the subgroups, we assessed heterogeneity of treatment effect with a test for interaction [51-55]. We estimated ORs along with 95\% CIs for each subgroup.

\section{Contamination}

To assess the potential for contamination, we report the proportion of control group participants that reported that they read another participant's messages and the proportion of intervention participants that reported that their messages were read by another participant.

\section{Change from baseline}

In addition to the analyses specified in the statistical analysis plan, we tested for a change in the primary outcome from baseline to follow-up, using McNemar's $x^{2}$ test for paired data. This post hoc non-randomized analysis was conducted to explore the increase in acceptability overall, as the app included intervention content (see Discussion).

\section{Results}

Recruitment, randomization, exclusions

Between 16 November 2016 and 1 March 2017, there were 580 randomizations. During the analysis, we discovered that five participants enrolled and were randomized twice. For the three participants that were allocated to the same arm on both randomizations, we kept them in the analysis using the baseline data from their first record. For the two participants that were allocated to different arms, we excluded them from the analysis. This resulted in 573 participants included in the trial (see Discussion).

Two hundred and seventy-five participants were allocated to the intervention arm and 298 participants were allocated to the control arm (Fig. 1). No participants withdrew from the trial after allocation.

\section{Baseline characteristics}

Baseline characteristics of trial participants are reported in Table 1. Mean age was 20 years, and 53\% were male. Ninety-four percent were not married (259/573), and only $2 \%(13 / 573)$ found at least one method of effective 


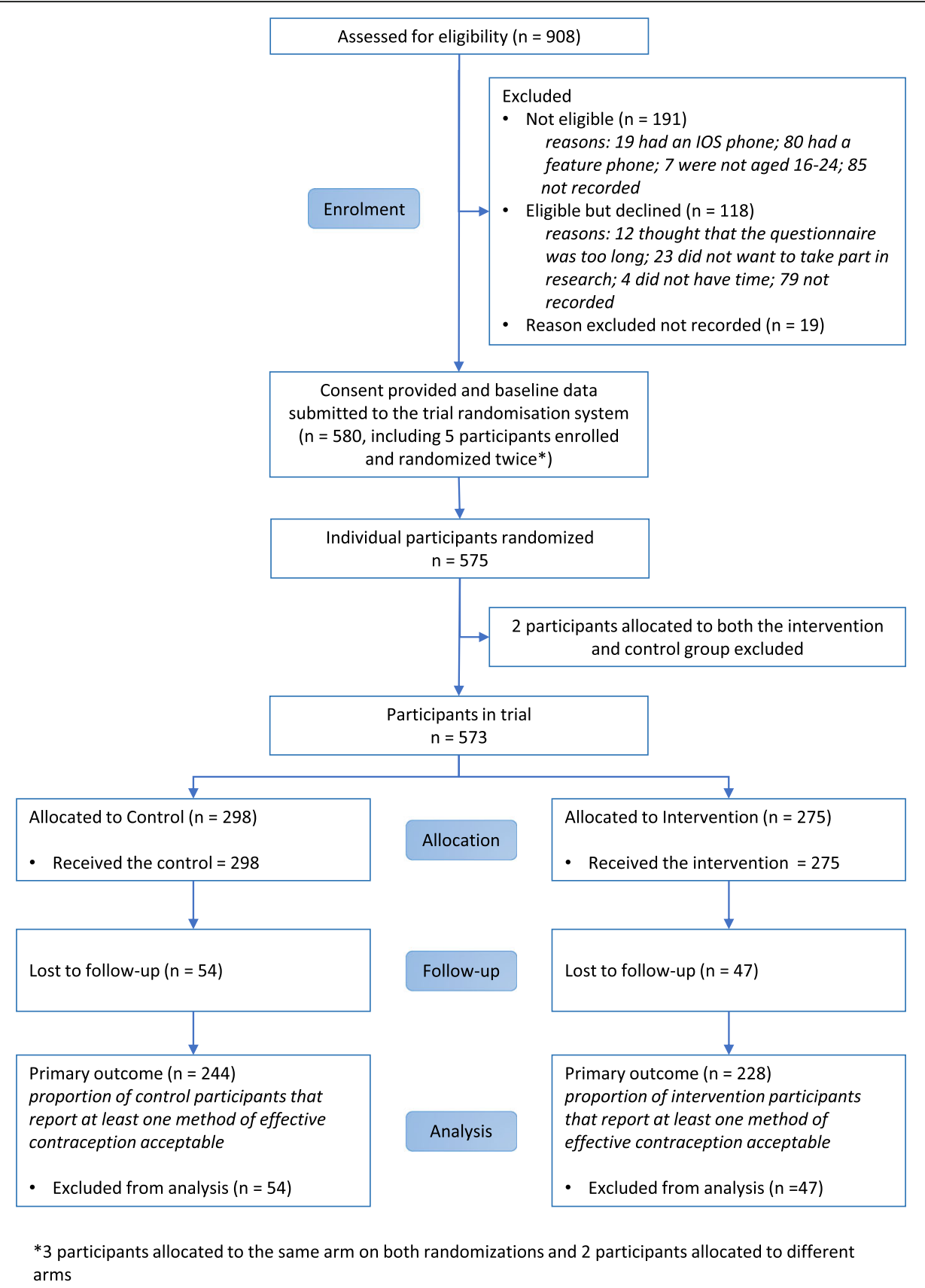

Fig. 1 CONSORT diagram

contraception acceptable. Characteristics were similar between the two groups.

\section{Loss to follow-up}

Four hundred and seventy-six participants total (83\%) contributed follow-up data. Four hundred and seventytwo participants $(82 \%)$ completed the trial follow-up for the primary outcome (intervention, $n=228$; control, $n=$ 244) (Fig. 1). Retention did not differ between the arms
(83\% in the intervention vs $82 \%$ in the control, $p=0.75$ ). The main predictors of retention were male gender (OR $1.78, p=0.01$ ), Tajik ethnicity (OR 2.22, $p=0.03$ ) and having completed a level of education lower than university at enrolment (OR 1.79, $p=0.02)$. The effect of these predictors did not differ by arm (interaction test $p$-values: gender, $p=0.72$; ethnicity, $p=0.41$; education level, $p=$ 0.98). Detailed characteristics of follow-up completers and non-completers are reported in Additional file 1. 
Table 1 Baseline characteristics

\begin{tabular}{|c|c|c|c|c|}
\hline & & $\begin{array}{l}\text { Control } \\
N=298, \% \\
\text { (n) }\end{array}$ & $\begin{array}{l}\text { Intervention } \\
N=275, \% \\
\text { (n) }\end{array}$ & $\begin{array}{l}\text { All } \\
\text { participants } \\
N=573, \% \\
\text { (n) }\end{array}$ \\
\hline \multirow[t]{3}{*}{$\overline{\text { Age }}$} & mean [sd] & $\begin{array}{l}20.00 \\
{[2.41]}\end{array}$ & $19.93[2.24]$ & $19.98[2.33]$ \\
\hline & $16-19$ & $53.02(158)$ & $56.73(156)$ & $54.80(314)$ \\
\hline & $20-24$ & $46.98(140)$ & 43.27(119) & $45.20(259)$ \\
\hline \multirow[t]{2}{*}{ Gender } & female & $45.97(137)$ & $47.27(130)$ & $46.60(267)$ \\
\hline & male & $54.03(161)$ & $52.73(145)$ & $53.40(306)$ \\
\hline \multirow[t]{2}{*}{ Marital status } & married & $6.71(20)$ & $5.82(16)$ & $6.28(36)$ \\
\hline & not-married & $93.29(278)$ & 94.18 (259) & $93.72(537)$ \\
\hline \multirow{3}{*}{$\begin{array}{l}\text { Number } \\
\text { of children }\end{array}$} & 0 & $95.64(285)$ & $97.09(267)$ & 96.34 (552) \\
\hline & 1 & $2.01(6)$ & $2.18(6)$ & $2.09(12)$ \\
\hline & 2 or more & $2.35(7)$ & $0.73(2)$ & $1.57(9)$ \\
\hline \multirow[t]{4}{*}{ Ethnicity } & Tajik & $92.62(276)$ & $93.82(258)$ & $93.19(534)$ \\
\hline & Russian & $2.35(7)$ & $0.36(1)$ & $1.40(8)$ \\
\hline & Uzbek & $5.03(15)$ & $5.45(15)$ & $5.24(30)$ \\
\hline & other & $0(0)$ & $0.36(1)$ & $0.17(1)$ \\
\hline \multirow[t]{7}{*}{ Occupation } & school & $17.79(53)$ & $17.09(47)$ & $17.45(100)$ \\
\hline & university & 68.46 (204) & 70.55 (194) & 69.46 (398) \\
\hline & working & $10.74(32)$ & 10.55 (29) & $10.65(61)$ \\
\hline & training & $0.67(2)$ & $0(0)$ & $0.35(2)$ \\
\hline & parent & $0.34(1)$ & $0(0)$ & $0.17(1)$ \\
\hline & not working & $1.68(5)$ & $1.82(5)$ & $1.75(10)$ \\
\hline & $\begin{array}{l}\text { university \& } \\
\text { working }\end{array}$ & $0.34(1)$ & $0(0)$ & $0.17(1)$ \\
\hline \multirow{4}{*}{$\begin{array}{l}\text { Highest level } \\
\text { of education } \\
\text { completed }\end{array}$} & primary & $12.75(38)$ & 13.09 (36) & $12.91(74)$ \\
\hline & secondary & $66.11(197)$ & $59.64(164)$ & $63.00(361)$ \\
\hline & university & $19.46(58)$ & $25.82(71)$ & $22.51(129)$ \\
\hline & other & $1.68(5)$ & $1.45(4)$ & $1.57(9)$ \\
\hline \multirow{4}{*}{$\begin{array}{l}\text { Current } \\
\text { pregnancy } \\
\text { intention } \\
\text { ('Do you want } \\
\text { a pregnancy } \\
\text { now?) }\end{array}$} & yes & $3.02(9)$ & $4.00(11)$ & $3.49(20)$ \\
\hline & no & $12.42(37)$ & $5.82(16)$ & $9.25(53)$ \\
\hline & unsure & $1.01(3)$ & $0.73(2)$ & $0.87(5)$ \\
\hline & not married ${ }^{a}$ & $83.56(249)$ & $89.45(246)$ & $86.39(495)$ \\
\hline \multirow[t]{6}{*}{ Baseline method } & none & $31.88(95)$ & $29.45(81)$ & $30.72(176)$ \\
\hline & $\begin{array}{l}\text { male } \\
\text { condom }\end{array}$ & $2.01(6)$ & $1.09(3)$ & $1.57(9)$ \\
\hline & $I U D^{b}$ & $0.67(2)$ & $0(0)$ & $0.35(2)$ \\
\hline & not married ${ }^{a}$ & $65.10(194)$ & $69.09(190)$ & $67.02(384)$ \\
\hline & $\operatorname{LAM}^{c}$ & $0(0)$ & $0.36(1)$ & $0.17(1)$ \\
\hline & other & $0.34(1)$ & $0(0)$ & $0.17(1)$ \\
\hline \multirow{2}{*}{$\begin{array}{l}\text { At least one } \\
\text { effective method } \\
\text { is acceptable }\end{array}$} & yes & $2.68(8)$ & $1.82(5)$ & $2.27(13)$ \\
\hline & no & $97.32(290)$ & $98.18(270)$ & $97.73(560)$ \\
\hline \multirow[t]{2}{*}{ Pill acceptability } & yes & $1.34(4)$ & $0.73(2)$ & $1.05(6)$ \\
\hline & no & 98.66 (294) & $99.27(273)$ & $98.95(567)$ \\
\hline IUD acceptability & yes & $1.34(4)$ & $0(0)$ & $0.70(4)$ \\
\hline
\end{tabular}

Table 1 Baseline characteristics (Continued)

\begin{tabular}{|c|c|c|c|c|}
\hline & & $\begin{array}{l}\text { Control } \\
N=298, \% \\
\text { (n) }\end{array}$ & $\begin{array}{l}\text { Intervention } \\
N=275, \% \\
\text { (n) }\end{array}$ & $\begin{array}{l}\text { All } \\
\text { participants } \\
N=573, \% \\
\text { (n) }\end{array}$ \\
\hline & no & 98.66 (294) & $100(275)$ & $99.30(569)$ \\
\hline \multirow{2}{*}{$\begin{array}{l}\text { Injection } \\
\text { acceptability }\end{array}$} & yes & $0.67(2)$ & $1.45(4)$ & $1.05(6)$ \\
\hline & no & 99.33 (296) & 98.55 (271) & 98.95 (567) \\
\hline \multirow{2}{*}{$\begin{array}{l}\text { Implant } \\
\text { acceptability }\end{array}$} & yes & $0.34(1)$ & $0.73(2)$ & $0.52(3)$ \\
\hline & no & $99.66(297)$ & $99.27(273)$ & $99.48(570)$ \\
\hline
\end{tabular}

aThe response 'not married' was used as a proxy for not being sexually active b/UD Intrauterine device

CAM Lactational amenorrhea method

\section{Primary outcome}

In the intervention arm, 66\% (151/228) reported that at least one method of contraception was acceptable compared to $64 \%(156 / 244)$ in the control arm (Table 2). There was no evidence of a difference in acceptability between the groups (crude OR 1.11, 95\% CI .76-1.62, $p=0.60$; adjusted OR 1.21, 95\% CI .80-1.83, $p=0.36$ ).

\section{Secondary outcomes}

There were no significant differences in any of the secondary outcomes between the groups (Table 3).

\section{Process outcomes}

There were no significant differences in any of the process outcomes between the groups (Table 4).

\section{Potential for contamination}

Three percent $(8 / 243)$ of control participants said that they read the messages of someone else in the study. Nine percent $(21 / 227)$ of intervention participants said that someone else in the study read their messages.

\section{Participants' report of physical violence during the study} Overall, $0.85 \%$ (4/470) reported that they experienced physical violence since being in the study $(0.41 \%$ in the control and $1.32 \%$ in the intervention, $p=0.57$ ).

\section{Sensitivity analyses}

The effect of the intervention on the primary outcome observed in the principal analysis did not change when we considered participants lost to follow-up did not find

Table 2 Primary outcome

\begin{tabular}{lllll}
\hline & $\begin{array}{l}\text { Control } \\
N=244, \% \\
(\mathrm{n})\end{array}$ & $\begin{array}{l}\text { Intervention } \\
\mathrm{N}=228, \%\end{array}$ & $\begin{array}{l}\text { OR } \\
(\mathrm{n})\end{array}$ & (95\% Cl) \\
\hline $\begin{array}{l}\text { At least one } \\
\text { effective method }\end{array}$ & $63.93(156)$ & $66.23(151)$ & $1.21(.80-1.83)$ & 0.36 \\
is acceptable & & & & \\
\hline
\end{tabular}

adjusted for pregnancy intention, gender, age, education level and acceptability at baseline 
Table 3 Secondary outcomes

\begin{tabular}{|c|c|c|c|c|}
\hline & Control \% (n/N) & Intervention \% (n/N) & OR $(95 \% \mathrm{Cl})$ & $p$-value \\
\hline Use of effective contraception ${ }^{a}$ & $3.66(9 / 246)$ & $1.30(3 / 230)$ & $.35(.06-1.42)$ & 0.18 \\
\hline Pill acceptability ${ }^{\mathrm{b}}$ & $56.56(138 / 244)$ & $60.53(138)$ & $1.32(.88-2.00)$ & 0.18 \\
\hline IUD acceptability ${ }^{\mathrm{b}}$ & $52.87(129 / 244)$ & $51.32(117 / 228)$ & $1.00(.67-1.50)$ & 0.98 \\
\hline Injection acceptability ${ }^{\mathrm{b}}$ & $54.51(133 / 244)$ & $55.26(126 / 228)$ & $1.14(.76-1.70)$ & 0.52 \\
\hline Implant acceptability ${ }^{\mathrm{b}}$ & $48.77(119 / 244)$ & $48.68(111 / 228)$ & $1.08(.73-1.59)$ & 0.71 \\
\hline Effective contraceptive use during the 4 months $^{a}$ & $2.88(7 / 243)$ & $1.76(4 / 227)$ & $.61(.13-2.42)$ & 0.62 \\
\hline Service uptake ${ }^{c}$ (attended a service one or more times) & $10.29(25 / 243)$ & $7.93(18 / 227)$ & $.76(.39-1.46)$ & 0.41 \\
\hline Unintended pregnancy ${ }^{c}$ & $0(0)$ & $0(0)$ & - & - \\
\hline Induced abortion ${ }^{c}$ & $0(0)$ & $0(0)$ & - & - \\
\hline
\end{tabular}

abased on unadjusted exact logistic regression, due to small numbers

badjusted for pregnancy intention, gender, age, education level and the corresponding method acceptability at baseline

cadjusted for pregnancy intention, gender, age, education level and acceptability at baseline

at least one method acceptable (OR 1.20, 95\% CI .84$1.73, p=0.31$ ) or when we adjusted the model for the predictors of missingness (OR 1.21, 95\% CI .80-1.85, $p=0.35$.

\section{Subgroup analysis}

There was some evidence that the effect of the intervention was greater among women compared to men (interaction test $p=0.03$ ). (Fig. 2).

\section{Change from baseline analysis}

Among the 472 participants who completed follow-up 2\% $(n=10)$ thought that at least one method was acceptable at baseline, which increased to $65 \%$ at follow-up $(n=307$, $p<0.001$ ) (Fig. 3). Acceptability for the individual methods increased from $1 \%$ at baseline to $49 \%-58 \%$ at follow-up $(p<0.001)$.

\section{Discussion}

\section{Main results}

Contrary to what was planned in the trial protocol, the app contained intervention content. Both intervention and control participants received intervention content targeting knowledge and attitudes towards effective contraception, including the BCM 'belief selection'. The trial therefore evaluated the effect of the whole intervention with all ten $\mathrm{BCMs}$ (belief selection, facilitation, anticipated regret, guided practice, verbal persuasion, tailoring, cultural similarity, arguments, shifting perspective and goal setting) delivered by instant messaging, compared to a proportion of the intervention delivered on the app pages with the $\mathrm{BCM}$ belief selection.

The trial found no evidence of a difference in acceptability of at least one effective contraceptive method between the intervention and control groups. There was also no evidence of a difference in any of the secondary and process outcomes between the groups (use of effective contraception, service uptake, knowledge, perceived norms, personal agency and intention to use effective contraception). This indicates that the intervention content delivered by the intervention messages only (includes nine additional BCMs targeting attitudes and personal agency) did not have an additional benefit over the app regarding these outcomes. The subgroup analysis suggests that the intervention delivered by instant messaging could be more effective among women compared to men. When data from both groups were analysed together, there was a large statistically significant increase in acceptability from baseline to follow-up.

\section{Comparisons with other research}

Trials that have evaluated interventions delivered by mobile phone to improve contraceptive-related outcomes have had mixed results [33-43]. We are conducting trials in Bolivia and Palestine that are evaluating the effect of interventions similar to the Tajik intervention on acceptability and use of effective contraception $[56,57]$. The results of the three trials together should contribute to a better understanding of the effect of the intervention evaluated in this Tajik trial.

Our trial shows no additional benefit on the outcomes from the nine BCMs deliver by instant messaging. No previous research reports the effectiveness of these $\mathrm{BCMs}$ aimed at improving contraceptive-related outcomes delivered by mobile phone [58].

Ongoing trials of interventions delivered by mobile phone to improve reproductive health are measuring participants' experience of violence during their participation in the trial $[56,57,59]$. In this Tajik trial, we found no association between the intervention and experience of violence. While this is reassuring, both groups had access to the app so we are unable to assess the effect of the app on partner violence. 
Table 4 Process outcomes

\begin{tabular}{|c|c|c|c|c|}
\hline & & Control \% (n/N) & Intervention \% (n/N) & $\begin{array}{l}\text { proportional OR }{ }^{*}(95 \% \mathrm{Cl}), \\
p \text {-value }\end{array}$ \\
\hline Knowledge of effective contraception & & $\begin{array}{l}\text { Mean }=4.00 \\
{[\mathrm{sd}=2.04]}\end{array}$ & $\begin{array}{l}\text { Mean }=4.08 \\
{[\mathrm{sd}=2.02]}\end{array}$ & $.08^{* *}(-.29-.44), 0.69$ \\
\hline \multirow{5}{*}{$\begin{array}{l}\text { My friends would use the pill, IUD, } \\
\text { injection or implant if they wanted } \\
\text { to prevent pregnancy }\end{array}$} & strongly disagree & $3.70(9 / 243)$ & $1.33(3 / 226)$ & \multirow[t]{5}{*}{$1.40(.97-2.01), 0.07$} \\
\hline & disagree & $4.53(11 / 243)$ & $5.31(12 / 226)$ & \\
\hline & not sure & $17.28(42 / 243)$ & $16.37(37 / 226)$ & \\
\hline & agree & $64.61(157 / 243)$ & $59.29(134 / 226)$ & \\
\hline & strongly agree & $9.88(24 / 243)$ & $17.70(40 / 226)$ & \\
\hline \multirow{5}{*}{$\begin{array}{l}\text { My friends would talk to their } \\
\text { husband/wife about contraception } \\
\text { if they wanted to prevent a pregnancy }\end{array}$} & strongly disagree & $1.23(3 / 243)$ & $1.33(3 / 226)$ & \multirow[t]{5}{*}{$1.09(.76-1.57), 0.64$} \\
\hline & disagree & $5.35(13 / 243)$ & $6.64(15 / 226)$ & \\
\hline & not sure & $16.05(39 / 243)$ & $15.93(36 / 226)$ & \\
\hline & agree & $65.02(158 / 243)$ & $59.29(134 / 226)$ & \\
\hline & strongly agree & $12.35(30 / 243)$ & $16.81(38 / 226)$ & \\
\hline \multirow{5}{*}{$\begin{array}{l}\text { If you wanted to use the pill, IUD, } \\
\text { injection or implant, how easy would } \\
\text { it be for you to use it? (women only) }\end{array}$} & very difficult & $7.62(8 / 105)$ & $5.83(6 / 103)$ & \multirow[t]{5}{*}{$1.43(.87-2.34), 0.16$} \\
\hline & difficult & $17.14(18 / 105)$ & $9.71(10 / 103)$ & \\
\hline & not sure & $27.62(29 / 105)$ & $29.13(30 / 103)$ & \\
\hline & easy & $38.10(40 / 105)$ & $43.69(45 / 103)$ & \\
\hline & very easy & $9.52(10 / 105)$ & $11.65(12 / 103)$ & \\
\hline \multirow{5}{*}{$\begin{array}{l}\text { If you wanted to talk to your husband/wife } \\
\text { about contraception, how easy would it be } \\
\text { for you to talk to him/her? }\end{array}$} & very difficult & $3.70(9 / 243)$ & $3 / 10(7 / 226)$ & \multirow[t]{5}{*}{$1.22(.86-1.73), 0.26$} \\
\hline & difficult & $6.17(15 / 243)$ & $7.52(17 / 226)$ & \\
\hline & not sure & $14.81(36 / 243)$ & $14.16(32 / 226)$ & \\
\hline & easy & $60.49(147 / 243)$ & $53.10(120 / 226)$ & \\
\hline & very easy & $14.81(36 / 243)$ & $22.12(50 / 226)$ & \\
\hline \multirow{5}{*}{$\begin{array}{l}\text { If you wanted to use the pill, IUD, } \\
\text { injection or implant, how certain are } \\
\text { you that you could use it? (women only) }\end{array}$} & very certain I could not & $2.86(3 / 105)$ & $5.83(6 / 103)$ & \multirow[t]{5}{*}{.99 (.60-1.63), 0.96} \\
\hline & certain I could not & $6.67(7 / 105)$ & $7.77(8 / 103)$ & \\
\hline & not sure & $38.10(40 / 105)$ & $32.04(33 / 103)$ & \\
\hline & certain I could & $40.00(42 / 105)$ & $41.75(43 / 103)$ & \\
\hline & very certain I could & $12.38(13 / 105)$ & $12.62(13 / 103)$ & \\
\hline \multirow{5}{*}{$\begin{array}{l}\text { If you wanted to talk to your husband/wife } \\
\text { about contraception, how certain are you } \\
\text { that you could talk to him/her? }\end{array}$} & very certain I could not & $1.23(3 / 243)$ & $2.65(6 / 226)$ & \multirow[t]{5}{*}{$1.10(.78-1.53), 0.60$} \\
\hline & certain I could not & $13.17(32 / 243)$ & $12.39(28 / 226)$ & \\
\hline & not sure & $16.46(40 / 243)$ & $16.81(38 / 226)$ & \\
\hline & certain I could & $50.62(123 / 243)$ & $44.25(100 / 226)$ & \\
\hline & very certain I could & $18.52(45 / 243)$ & $23.89(54 / 226)$ & \\
\hline \multirow{5}{*}{$\begin{array}{l}\text { I intend to use the pill, IUD, } \\
\text { injection or implant }\end{array}$} & strongly disagree & $4.76(5 / 105)$ & $2.91(3 / 103)$ & \multirow[t]{5}{*}{$1.37(.84-2.25), 0.21$} \\
\hline & disagree & $10.48(11 / 105)$ & $12.62(13 / 103)$ & \\
\hline & not sure & $31.43(33 / 105)$ & $25.24(26 / 103)$ & \\
\hline & agree & $39.05(41 / 105)$ & $34.95(36 / 103)$ & \\
\hline & strongly agree & $14.29(15 / 105)$ & $24.27(25 / 103)$ & \\
\hline \multirow[t]{4}{*}{ Number of messages read } & all & & $32.16(73 / 227)$ & \\
\hline & most & & $43.61(99 / 227)$ & \\
\hline & some & & $18.50(42 / 227)$ & \\
\hline & none & & $5.73(13 / 227)$ & \\
\hline $\begin{array}{l}\text { Proportion of intervention participants } \\
\text { that stopped the intervention }\end{array}$ & & & $29.07(66 / 227)$ & \\
\hline
\end{tabular}




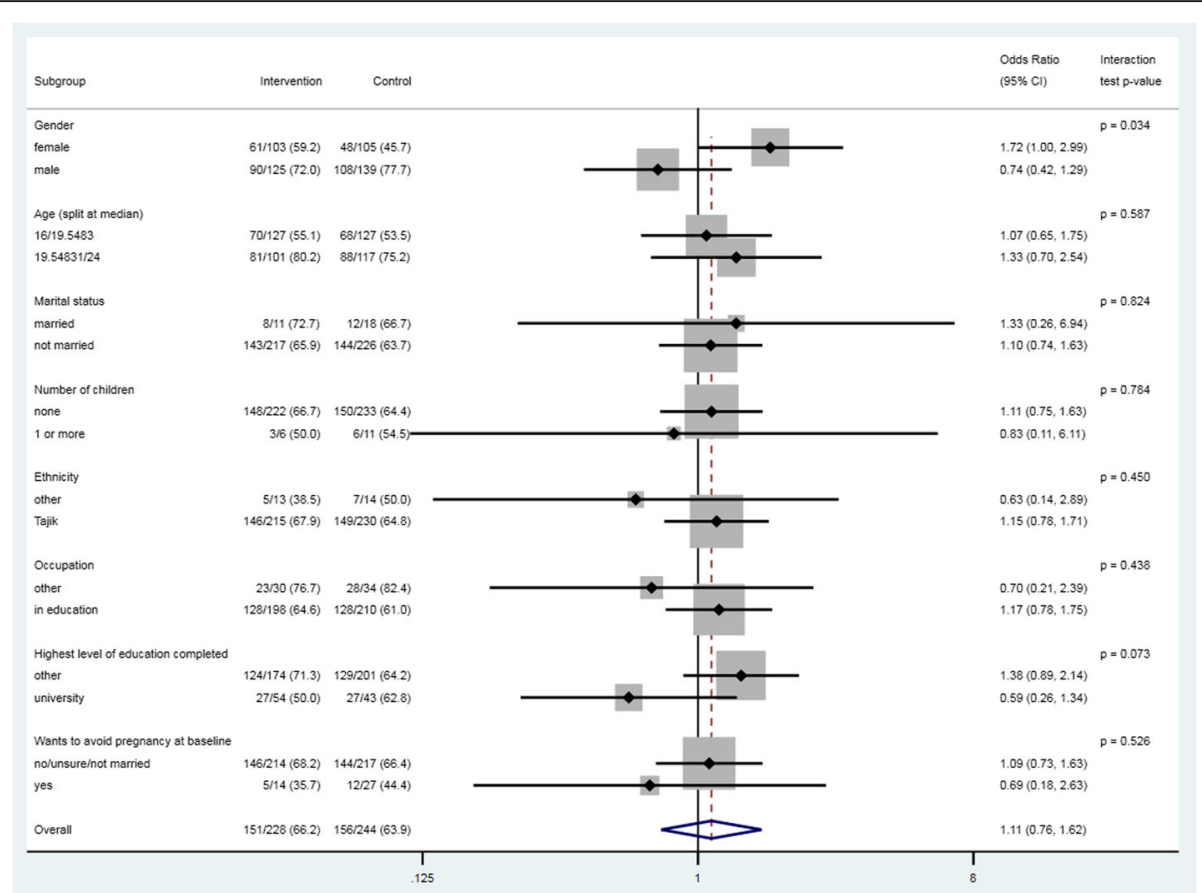

Fig. 2 Primary outcome by pre-specified subgroups

\section{Strengths and limitations}

The trial conduct has a number of strengths. We recruited our target number of participants and were able to collect follow-up data for an acceptable proportion of them, given that the sample size allowed for $20 \%$ loss. We developed and tested a remote trial database and randomization system, which successfully generated and concealed the allocation sequence and achieved well-balanced groups. An important limitation is that the app included intervention content, as discussed above. This constitutes a protocol deviation and the trial was therefore not able to answer the primary question it aimed to answer. Because the selfreported acceptability scales were collected by telephone by the research staff, participants may have been more likely to report positive attitudes than they were at baseline where they completed the questionnaire by themselves on their phones. Regarding the large increase in acceptability from baseline to follow-up, we cannot rule out the possibility that at least a portion of this increase was due to participation in the trial as opposed to the intervention itself; participants were aware that the trial involved changing attitudes towards contraception. Five participants enrolled and were randomized twice.

There were inconsistencies in participants' selfreporting of marital status. The proportion that responded 'not married' to the current pregnancy intention $(495 / 573,86 \%)$ and the baseline method question $(384 / 573,67 \%)$ is lower than the proportion that responded 'not married' when asked directly about their marital status $(537 / 573,94 \%)$. We cannot say why

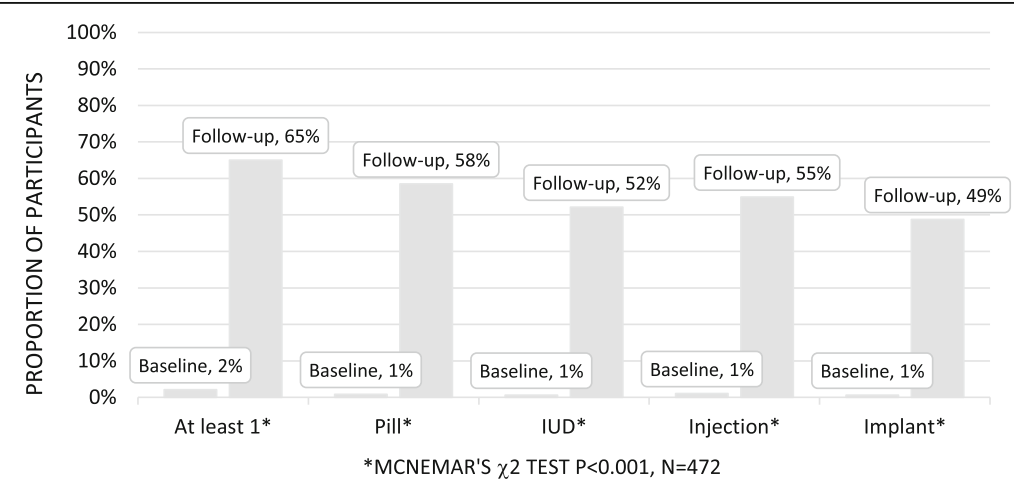

Fig. 3 Method acceptability at baseline and follow-up 
these inconsistencies occurred. However, we can speculate that some participants who responded 'not married' to the marital status question were sexually active and responded to the other two questions with responses other than 'not married'.

Thirty six percent of people assessed for eligibility (328/908) were excluded from the study. The reason for ineligibility was not recorded for 85 people, which could limit the generalizability of the trial findings. While the recording of this information was not complete, of those that are known, the majority appear to have been excluded because they either did not have an Android phone $(n=99)$. If those who do not own a smartphone are less likely to find at least one method of effective contraception acceptable, this could affect the generalisability of the results. Smartphone ownership is rapidly increasing however, and ownership could be an option for a greater proportion of young people across different socioeconomic communities in the near future.

\section{Implications of the findings}

The finding that the intervention instant messages did not have an additional benefit over the app along with the large increase in acceptability from baseline to follow-up suggests that participants read the app contraception pages. It may be that in a context such as Tajikistan, where young people have limited access to information and support about reproductive health, they are willing to read static app pages about this topic. In comparison, a trial in the United Kingdom found that young people did not engage heavily with a sexual and reproductive health website $[60,61]$. In contexts such as the United Kingdom where information and support are more accessible, interventions delivered on app pages and websites may be utilized less frequently than in contexts such as Tajikistan.

Because the intervention content included on the app aimed to improve knowledge of and attitudes towards effective contraception, it is not surprising that there was no evidence of a difference between the groups regarding these outcomes. Though the large increase in acceptability from baseline to follow-up cannot be unequivocally attributed to the intervention content, an increase this large suggests that the intervention content included on the app at least was partially effective in improving attitudes towards the effective methods. Because the intervention is well-specified, we were able to identify the components of the intervention that may have been effective in producing this change (accurate information and targeting beliefs using the $\mathrm{BCM}$ belief selection) [46, 48].
Despite the contamination that occurred, intervention participants received content that control participants did not. The secondary outcomes use and service uptake and the process outcomes personal agency and intention are related to the content that only intervention participants received. There are a number of potential explanations for why we did not observe a difference between the groups in these outcomes. The first is that the BCMs targeting these outcomes did not work. This could have been because the conditions under which the methods have been shown to be effective were not fully satisfied $[46,48]$. In addition, because a large proportion of meaning comes from visual cues in face-to-face interaction [46], some of the meaning of the BCMs may have been lost when delivered by mobile phone. For example, the BCM 'guided practice' requires skill demonstration, enactment and individual feedback. While the intervention messages demonstrated and provided instruction, we were not able to observe the participant enacting the behavior or to provide individual feedback. This may have resulted in a loss of effectiveness of the BCM. Another explanation is that intervention could be more effective on these secondary and process outcomes with people where the behaviour is salient, such as with those who are married/sexually active or soon to be. In this trial however, only $6 \%(36 / 573)$ were married/sexually active, which was too small to explore this possibility. Alternatively, the app alone may have been effective in influencing these secondary and process outcomes; in the Tajik context, providing accurate information from a credible source and targeting the preidentified beliefs may be sufficient. Finally, these secondary and process outcomes could have be so strongly influenced by environmental conditions (e.g. stigma regarding sexual activity before marriage and pressure to bear children) that they are not amenable to change by a mobile phone intervention only.

While caution is necessary in interpreting the results of the subgroup analysis, it suggests that the whole intervention delivered by instant messaging could be more effective among women compared to men. The trials in Bolivia and Palestine involve women only so the results should provide additional evidence of the intervention's effectiveness in women.

We are currently conducting qualitative interviews with trial participants to explore their experiences in receiving the intervention and app content. If participants were positive about receiving the intervention messages, this could support the delivery of the messages with the download of the app. The fact that the intervention is already developed and therefore inexpensive to deliver, plus the fact that it does not appear to cause harm, also supports the delivery of the messages with the download of the app. 


\section{Conclusions}

This trial demonstrated that the whole intervention delivered by app instant messaging provided no additional benefit over a portion of the intervention delivered by the app pages. An analysis of participants randomized to the control and intervention groups together showed a large significant increase in acceptability from baseline to follow-up. Further research is needed to establish the effect of the intervention on attitudes towards and use of effective contraceptive methods among married/sexually active young people.

\section{Additional file}

Additional file 1 Baseline data by follow-up status. Baseline characteristics by follow-up completion. The baseline characteristics of the participants that completed the primary outcome and the baseline characteristics of participants that did not complete the primary outcome. (DOCX $17 \mathrm{~kb}$ )

\section{Abbreviations}

BCM: Behaviour change method; Cl: Confidence interval; IPPF: International Planned Parenthood Federation; LSHTM: London School of Hygiene \& Tropical Medicine; OR: Odds ratio; TFPA: Tajik Family Planning Association

\section{Acknowledgements}

We would like to thank the young people in Tajikistan who participated in both the intervention development phase and the trial. We would also like to thank Matlyuba Salikhova, Safarbek Saidov, Ramziyor Saydaliev and Khusrav Bobobekov who promoted the study and assisted with collection of the follow-up data. We are extremely grateful for their hard work and volunteering their time. Finally, we would like to thank the International Planned Parenthood Federation for funding the trial and Alison McKinley, the Senior Research and Evaluation Officer for the Innovation Programme, for her support throughout the project.

\section{Funding}

The trial was supported by the International Planned Parenthood Federation Innovation Programme. IPPF had some influence over the study design (MV and SS) but were not involved in the data collection or analysis.

\section{Availability of data and materials}

The anonymised dataset used and analysed for the current study are available from the corresponding author.

\section{Authors' contributions}

OLM designed and managed the trial, conducted the analysis and wrote the manuscript. FK coordinated and conducted the trial recruitment and follow-up and contributed to planning discussions regarding the trial. RT facilitated trial implementation, contributed to planning discussions regarding the trial and took overall local responsibility for the project. SSa facilitated trial implementation and contributed to planning discussions regarding the trial. MV and SSt contributed to planning discussions regarding the trial. IA developed the trial database and randomization system. BL provided advice regarding the statistical analysis and reviewed the Stata analysis code. PE provided oversight regarding the statistical analysis. MP reviewed the primary outcome Stata analysis code. CF provided guidance regarding the trial design and took overall academic responsibility for the project. All authors revised the work, approved the version to be published and agree to be accountable for all aspects of the work.

\section{Ethics approval and consent to participate}

Ethical approval was granted from the London School of Hygiene and Tropical Medicine Observational Research Ethics Committee on 27 April 2015 (reference number 9148), the Tajik National Scientific and Research Centre on Pediatrics and Child Surgery on 8 June 2015.
All participants provided informed consent before providing baseline data and randomization.

\section{Consent for publication}

Not applicable

\section{Competing interests}

The authors declare that they have no competing interests.

\section{Publisher's Note}

Springer Nature remains neutral with regard to jurisdictional claims in published maps and institutional affiliations.

\section{Author details}

'Department of Population Health, Faculty of Epidemiology and Population Health, London School of Hygiene \& Tropical Medicine, Keppel Street, London WC1E 7HT, UK. ${ }^{2}$ Tajik Family Planning Association, 10 Rudaki Avenue, TC 'Sadbarg', 7th floor, Dushanbe, Tajikistan. ${ }^{3}$ International Planned Parenthood Federation European Network, Rue Royale 146, 1000 Brussels, Belgium. ${ }^{4}$ Department of Medical Statistics, Faculty of Epidemiology and Population Health, London School of Hygiene \& Tropical Medicine, Keppel Street, London WC1E 7HT, UK.

Received: 20 October 2017 Accepted: 5 February 2018

Published online: 13 February 2018

\section{References}

1. Sedgh $G$, Singh $S$, Hussain R. Intended and unintended pregnancies worldwide in 2012 and recent trends. Stud Fam Plan. 2014;45(3):301-14.

2. Tsui $A O$, McDonald-Mosley $R$, Burke AE. Family planning and the burden of unintended pregnancies. Epidemiol Rev. 2010;32:152-74.

3. Logan C, Holcombe E, Manlove J, Ryan S. The consequences of unintended childbearing: a white paper. Washington, DC: Child Trends; 2007.

4. Khajehpour M, Simbar M, Jannesari S, Ramezani-Tehrani F, Majd HA. Health status of women with intended and unintended pregnancies. Public Health. 2013:127(1):58-64

5. Gipson JD, Koenig MA, Hindin MJ. The effects of unintended pregnancy on infant, child, and parental health: a review of the literature. Stud Fam Plan 2008;39(1):18-38.

6. Nakku JEM, Nakasi G, Mirembe F. Postpartum major depression at six weeks in primary health care: prevalence and associated factors. Afr Health Sci. 2006;6(4):207-14.

7. Cheng D, Schwarz EB, Douglas E, Horon I. Unintended pregnancy and associated maternal preconception, prenatal and postpartum behaviors. Contraception 2009:79(3):194-8.

8. Marston C, Cleland J. Do unintended pregnancies carried to term lead to adverse outcomes for mother and child? An assessment in five developing countries. Popul Stud. 2003;57(1):77-93.

9. Shah PS, Balkhair T, Ohlsson A, Beyene J, Scott F, Frick C. Intention to become pregnant and low birth weight and preterm birth: a systematic review. Matern Child Health J. 2011;15(2):205-16.

10. Mohllajee AP, Curtis KM, Morrow B, Marchbanks PA. Pregnancy intention and its relationship to birth and maternal outcomes. Obstet Gynecol. 2007; 109(3):678-86.

11. Brown SS, Eisenberg $L$, editors. The Best Intentions: Unintended Pregnancy and the Well-Being of Children and Families. Washington D.C.: National Academies Press; 1995

12. Darroch JE, Audam S, Biddlecom A, Kopplin G, Riley T, Singh S, et al. Adding it up: the costs and benefits of investing in sexual and reproductive health 2017 (Investing in contraception and maternal and newborn health fact sheet): Guttmacher Institute; 2017.

13. Singh S, Darroch J, Ashford L. Adding it up: the costs and benefits of investing in sexual and reproductive health 2014. New York: Guttmacher Institute; 2014

14. UNFPA Tajikistan country Programme 2010-2015 evaluation report. United Nations Population Fund; 2014

15. Country programme document for Tajikistan. United Nations Population Fund; 2015

16. Tajikistan Demographic and Health Survey 2012. Dushanbe, Tajikistan, and Calverton, Maryland, USA: SA, MOH, and ICF International.: Statistical Agency 
under the President of the Republic of Tajikistan (SA), Ministry of Health [Tajikistan], and ICF International., 2013.

17. Cleland J, Ali MM. Reproductive consequences of contraceptive failure in 19 developing countries. Obstet Gynecol. 2004;104(2):314-20.

18. Trussell J. Contraceptive efficacy. In: Hatcher R, Trussell J, Nelson A, Cates W, Kowal D, Policar M, editors. Contraceptive technology: twentieth revised edition. 20th ed. New York: Ardent Media; 2011.

19. Trussell J. Contraceptive efficacy: global library of women's medicine; 2014 [26 September 2017]. Available from: http://www.glowm.com/section_view/ heading/Contraceptive\%20Efficacy/item/374.

20. Sedgh G, Ashford L, Hussain R. Unmet need for contraception in developing countries: examining Women's reasons for not using a method. New York: Guttmacher Institute; 2016.

21. Free $C$, Knight $R$, Robertson $S$, Whittaker R, Edwards $P$, Zhou W, et al. Smoking cessation support delivered via mobile phone text messaging (txt2stop): a single-blind, randomised trial. Lancet. 2011;378(9785):49-55.

22. Lester RT, Ritvo P, Mills EJ, Kariri A, Karanja S, Chung MH, et al. Effects of a mobile phone short message service on antiretroviral treatment adherence in Kenya (WelTel Kenya1): a randomised trial. Lancet. 2010;376(9755):1838-45.

23. Pop-Eleches C, Thirumurthy H, Habyarimana JP, Zivin JG, Goldstein MP, de Walque $D$, et al. Mobile phone technologies improve adherence to antiretroviral treatment in a resource-limited setting: a randomized controlled trial of text message reminders. AIDS. 2011;25(6):825-34.

24. Zurovac D, Sudoi RK, Akhwale WS, Ndiritu M, Hamer DH, Rowe AK, et al. The effect of mobile phone text-message reminders on Kenyan health workers' adherence to malaria treatment guidelines: a cluster randomised trial. Lancet. 2011;378(9793):795-803.

25. Orr JA, King RJ. Mobile phone SMS messages can enhance healthy behaviour: a meta-analysis of randomised controlled trials. Health Psychol Rev. 2015;9(4):397-416.

26. Hall CS, Fottrell E, Wilkinson S, Byass P. Assessing the impact of mHealth interventions in low- and middle-income countries-what has been shown to work? Glob Health Action. 2014;7:25606.

27. Aranda-Jan CB, Mohutsiwa-Dibe N, Loukanova S. Systematic review on what works, what does not work and why of implementation of mobile health (mHealth) projects in Africa. BMC Public Health. 2014;14:188.

28. Free C, Phillips G, Galli L, Watson L, Felix L, Edwards P, et al. The effectiveness of mobile-health technology-based health behaviour change or disease management interventions for health care consumers: a systematic review. PLoS Med. 2013;10(1):e1001362.

29. Head KJ, Noar SM, lannarino NT, Grant Harrington N. Efficacy of text messaging-based interventions for health promotion: a meta-analysis. Soc Sci Med. 2013;97:41-8.

30. Hall AK, Cole-Lewis H, Bernhardt JM. Mobile text messaging for health: a systematic review of reviews. Annu Rev Public Health. 2015;36:393-415.

31. L'Engle KL, Mangone ER, Parcesepe AM, Agarwal S, Ippoliti NB. Mobile phone interventions for adolescent sexual and reproductive health: a systematic review. Pediatrics. 2016;138(3):e20160884.

32. Ippoliti NB, L'Engle KL. Meet us on the phone: mobile phone programs for adolescent sexual and reproductive health in low-to-middle income countries. Reprod Health. 2017;14(1):11.

33. Berenson AB, Rahman M. A randomized controlled study of two educational interventions on adherence with oral contraceptives and condoms. Contraception. 2012;86(6):716-24.

34. Castaño PM, Bynum JY, Andres R, Lara M, Westhoff C. Effect of daily text messages on oral contraceptive continuation: a randomized controlled trial. Obstet Gynecol. 2012;119(1):14-20.

35. Trent M, Thompson C, Tomaszewski K. Text messaging support for urban adolescents and young adults using injectable contraception: outcomes of the DepoText pilot trial. J Adolesc Health. 2015;57(1):100-6.

36. Smith C, Ngo TD, Gold J, Edwards P, Vannak U, Sokhey L, et al. Effect of a mobile phone-based intervention on post-abortion contraception: a randomized controlled trial in Cambodia. Bull World Health Organ. 2015;

37. Hall KS, Westhoff CL, Castano PM. The impact of an educational text message intervention on young urban women's knowledge of oral contraception. Contraception. 2013;87(4):449-54.

38. Rokicki S, Cohen J, Salomon JA, Fink G. Impact of a text-messaging program on adolescent reproductive health: a cluster-randomized trial in Ghana. Am J Public Health. 2017;107(2):298-305.

39. Johnson D, Juras R, Riley P, Chatterii M, Sloane P, Choi SK, et al. A randomized controlled trial of the impact of a family planning mHealth service on knowledge and use of contraception. Contraception. 2017;95(1): 90-7.

40. Tsur L, Kozer E, Berkovitch M. The effect of drug consultation center guidance on contraceptive use among women using isotretinoin: a randomized, controlled study. J Womens Health (Larchmt). 2008;17(4):57984.

41. Hou MY, Hurwitz S, Kavanagh E, Fortin J, Goldberg AB. Using daily textmessage reminders to improve adherence with oral contraceptives: a randomized controlled trial.[erratum appears in Obstet Gynecol. 2010 Nov; 116(5):1224]. Obstet Gynecol. 2010;116(3):633-40.

42. Kirby D, Raine T, Thrush G, Yuen C, Sokoloff A, Potter SC. Impact of an intervention to improve contraceptive use through follow-up phone calls to female adolescent clinic patients. Perspect Sex Reprod Health. 2010;42(4): 251-7.

43. Bull S, Devine S, Schmiege SJ, Pickard L, Campbell J, Shlay JC. Text messaging, teen outreach program, and sexual health behavior: a cluster randomized trial. Am J Public Health. 2016;106(S1):S117-S24.

44. McCarthy OL, Leurent B, Edwards P, Tokhirov R, Free C. A randomised controlled trial of an intervention delivered by app instant messaging to increase the acceptability of effective contraception among young people in Tajikistan: study protocol. BMJ Open. 2017;7(9):e017606.

45. McCarthy OL. A randomised controlled trial of an intervention delivered by app instant messaging to increase the acceptability of effective contraception among young people in Tajikistan: statistical analysis plan. Figshare. https://doi.org/10.6084/m9.figshare.5314714.v2.

46. Bartholomew Eldredge LK, Markham C, Ruiter R, Fernandez M, Kok G, Parcel G. Planning health promotion programs: an intervention mapping approach. 4th ed. San Francisco: Jossey-Bass; 2016.

47. Montaño D, Kasprzyk D. Theory of reasoned action, theory of planned behavior, and the integrated behavioral model. In: Glanz K, Rimer BK, Viswanath K, editors. Health behaviour: theory, research and practice. 5th ed. San Francisco: Jossey-Bass; 2015. p. 168-222.

48. Kok G, Gottlieb N, Peters G, Mullen P, Parcel G, Ruiter R, et al. A Taxonomy of Behavior Change Methods; an Intervention Mapping Approach. Health Psychol Rev. 2016;10(3):297-12.

49. Francis JJ, Eccles MP, Johnston M, Walker A, Grimshaw J, Foy R, et al. Constructing questionnaires based on the theory of planned behaviour. A manual for health services researchers 2004; 2010. p. 2-12.

50. Montano DE, Kasprzyk D, Hamilton DT, Tshimanga M, Gorn G. Evidencebased identification of key beliefs explaining adult male circumcision motivation in Zimbabwe: targets for behavior change messaging. AIDS Behav. 2014;18(5):885-904.

51. Pocock SJ, Assmann SE, Enos LE, Kasten LE. Subgroup analysis, covariate adjustment and baseline comparisons in clinical trial reporting: current practice and problems. Stat Med. 2002;21 (19):2917-30.

52. Kasenda B, Schandelmaier S, Sun X, von Elm E, You J, Blumle A, et al. Subgroup analyses in randomised controlled trials: cohort study on trial protocols and journal publications. BMJ. 2014;349:94539.

53. Gabler NB, Duan N, Liao D, Elmore JG, Ganiats TG, Kravitz RL. Dealing with heterogeneity of treatment effects: is the literature up to the challenge? Trials. 2009;10:43.

54. Kent DM, Rothwell PM, loannidis JPA, Altman DG, Hayward RA. Assessing and reporting heterogeneity in treatment effects in clinical trials: a proposal. Trials. 2010;11:85.

55. Brookes ST, Whitely E, Egger M, Smith GD, Mulheran PA, Peters TJ. Subgroup analyses in randomized trials: risks of subgroup-specific analyses; power and sample size for the interaction test. J Clin Epidemiol. 2004;57(3):229-36.

56. McCarthy OL, Wazwaz O, Jado I, Leurent B, Edwards P, Adada S, et al. An intervention delivered by text message to increase the acceptability of effective contraception among young women in Palestine: study protocol for a randomised controlled trial. Trials. 2017;18(1):454.

57. McCarthy OL, Osorio Calderon V, Makleff S, Huaynoca S, Leurent B, Edwards P, et al. An intervention delivered by app instant messaging to increase acceptability and use of effective contraception among young women in Bolivia: protocol of a randomized controlled trial. JMIR Res Protoc. 2017;6(12):e252.

58. Smith C, Gold J, Ngo TD, Sumpter C, Free C. Mobile phone-based interventions for improving contraception use. Cochrane Database Syst Rev. 2015;(6):CD011159.

59. Reiss K, Andersen K, Barnard S, Ngo TD, Biswas K, Smith C, et al. Using automated voice messages linked to telephone counselling to increase post-menstrual regulation contraceptive uptake and continuation in 
Bangladesh: study protocol for a randomised controlled trial. BMC Public Health. 2017:17(1):769.

60. Bailey JV, Pavlou M, Copas A, McCarthy O, Carswell K, Rait G, et al. The sexunzipped trial: optimizing the design of online randomized controlled trials. J Med Internet Res. 2013;15(12):e278.

61. Carswell K, McCarthy O, Murray E, Bailey JV. Integrating psychological theory into the design of an online intervention for sexual health: the sexunzipped website. JMIR Res Protoc. 2012;1(2):e16.

Submit your next manuscript to BioMed Central and we will help you at every step:

- We accept pre-submission inquiries

- Our selector tool helps you to find the most relevant journal

- We provide round the clock customer support

- Convenient online submission

- Thorough peer review

- Inclusion in PubMed and all major indexing services

- Maximum visibility for your research

Submit your manuscript at www.biomedcentral.com/submit 